\title{
Uplink Multi-Cell Processing: Approximate Sum Capacity under a Sum Backhaul Constraint
}

\author{
Yuhan Zhou*, Wei Yu*, and Dimitris Toumpakaris ${ }^{\dagger}$ \\ *Department of Electrical and Computer Engineering, University of Toronto, Canada \\ ${ }^{\dagger}$ Department of Electrical and Computer Engineering, University of Patras, Greece \\ Emails: \{yzhou,weiyu\}@comm.utoronto.ca,dtouba@upatras.gr
}

\begin{abstract}
This paper investigates an uplink multi-cell processing (MCP) model where the cell sites are linked to a central processor (CP) via noiseless backhaul links with limited capacity. A simple compress-and-forward scheme is employed, where the base-stations (BSs) quantize the received signals and send the quantized signals to the $C P$ using distributed Wyner-Ziv compression. The $\mathrm{CP}$ decodes the quantization codewords first, then decodes the user messages as if the users and the $\mathrm{CP}$ form a virtual multiple-access channel. This paper formulates the problem of maximizing the overall sum rate under a sum backhaul constraint for such a setting. It is shown that setting the quantization noise levels to be uniform across the BSs maximizes the achievable sum rate under high signal-to-noise ratio (SNR). Further, for general SNR a low-complexity fixed-point iteration algorithm is proposed to optimize the quantization noise levels. This paper further shows that with uniform quantization noise levels, the compress-and-forward scheme with Wyner-Ziv compression already achieves a sum rate that is within a constant gap to the sum capacity of the uplink MCP model. The gap depends linearly on the number of BSs in the network but is independent of the SNR and the channel matrix.
\end{abstract}

\section{INTRODUCTION}

Inter-cell interference is a fundamental bottleneck in wireless cellular networks. A promising technique for inter-cell interference mitigation is joint multi-cell processing (MCP) in which the transmitted and received signals and the channel state information are shared among the base-stations (BSs). By taking advantage of the high-capacity backhaul links, a network employing MCP can jointly encode or decode messages from multiple cells [1], [2].

This paper studies the uplink of a MCP model where the BSs are connected to a central processor (CP) via noiseless backhaul links with finite sum capacity $C$ as shown in Fig. 1. This uplink MCP model can be thought of as a virtual multiple-access channel (V-MAC) between the users and the $\mathrm{CP}$, with the BSs acting as relays. This paper utilizes a simple compress-and-forward strategy, in which the BSs quantize the received signals using Wyner-Ziv coding and then forward the quantized bits to the $\mathrm{CP}$. The $\mathrm{CP}$ first obtains the quantized signals from the quantized bits, then decodes the user messages based on the quantized signals. Our contributions are two-fold: First, we show that this simple version of the compress-andforward scheme already achieves the sum capacity of uplink MCP under a sum backhaul constraint to within a constant gap, thereby leading to a simple receiver architecture for the uplink MCP model. Second, we optimize the quantization

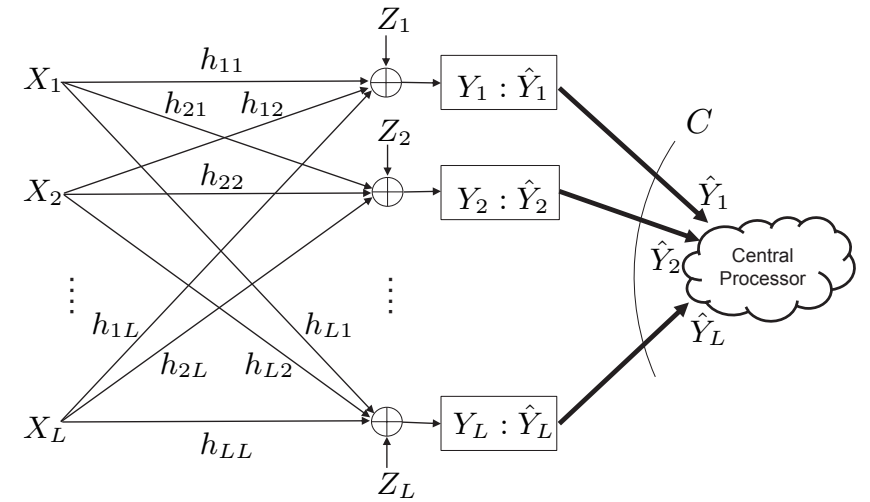

Fig. 1. Uplink multicell joint processing via a central processor

noise levels to maximize the sum rate for this MCP model. Our main insight is that setting the quantization noise levels to be uniform across the BSs is asymptotically optimal under high SNR, and also achieves constant gap to sum capacity under arbitrary SNR.

The limited backhaul MCP model has been studied in the literature. Specifically, [3], [4], [5] consider a similar model in which multiple users (or a single user with multiple antennas) communicate with a remote destination via multiple relays and derive achievable rates based on the compressand-forward and other relaying schemes. The achievable rate studied in this paper is similar to that of [3, Theorem 1], but we go further in explicitly optimizing the quantization levels, which allows us to characterize the approximate sum capacity of the overall system. This paper differs from [4], [5] in that [4], [5] mainly focus on a more general achievability scheme in which the quantized signals and the transmitted signals are decoded jointly, while this paper shows that one can decode the quantized signals and the transmitted signals successively without loss of approximate optimality in sum capacity. Our result depends on the fact that we consider a sum-capacity constraint in the backhaul, as opposed to the individual backhaul constraints considered in [3], [4], [5]. The proof relies on a judicious choice of quantization noise levels and a comparison with a cut-set like upper bound.

It is worth noting that the achievability schemes of [4], [5] and [3, Corollary 1] can be thought of as a particular instance of the noisy network coding scheme [6], [7], and the uplink MCP model is an example of a multi-message multicast relay network. Noisy network coding achieves the 
capacity region of arbitrary multicast relay networks to within a constant gap. Consequently, the achievability scheme of [4], [5] and [3, Corollary 1] must already achieve the capacity region of the uplink MCP model to within a constant gap. However, the constant-gap results of [6], [7] are possible only if the quantization noise levels are set to be at the background noise level, and only if joint decoding of the quantized signals and the transmitted signals is performed. Such joint decoding is prohibitively complex. In fact, even a mere evaluation of the rate region that is achievable by noisy network coding already involves $2^{L}-1$ constraints, each of which is a minimization over $2^{L}$ terms. One of the main points of this paper is that such an exponential complexity can be avoided and successive decoding is already optimal to within a constant gap, if we focus on the sum capacity under a sum backhaul constraint.

A main focus of this paper is the optimization of the quantization noise levels at the BSs. Such an optimization is recognized as a difficult nonconvex problem. Various algorithms have been proposed in the literature from, e.g., capacity maximization [8] and robustness [9] points of view. This paper focuses on the case where each BS is equipped with only a single antenna, (in contrast to the MIMO case treated in [8].) We point out that to maximize the achievable sum rate under a sum backhaul constraint, the quantization noise levels can be set to be uniform. This can be justified both under a high SNR assumption, and by examining the sum capacity outer bound. This paper further proposes a fixed-point iteration algorithm to find the optimal quantization noise levels across all the BSs at practical SNRs. The proposed technique optimizes the quantization noise levels across all the BSs jointly, whereas in [8] the optimization is done sequentially for each BS.

\section{Channel Model}

We consider the uplink of a multi-cell network where $L$ single-antenna mobile users send independent messages to $L$ single-antenna BSs, as shown in Fig. 1. The BSs are connected to a $\mathrm{CP}$ through noiseless backhaul links of capacities $C_{i}$, $i=1, \ldots, L$. The $C_{i}$ 's need to satisfy a sum capacity constraint: $\sum_{i=1}^{L} C_{i} \leq C$. This sum backhaul constraint can model the case where the backhaul is implemented by wireless links operating in a time- or frequency-division multiplexing basis and under power spectrum density constraint. The user messages need to be eventually decoded at the CP.

The uplink MCP model can be thought of as a virtual multiple-access channel between the users and the $\mathrm{CP}$ with the BSs serving as relay nodes. Let $X_{i}$ denote the signal transmitted by the $i$-th user. Then the signal received at the $i$-th BS can be expressed as

$$
Y_{i}=\sum_{j=1}^{L} h_{i j} X_{j}+Z_{i} \text { for } i=1,2, \ldots, L,
$$

where $Z_{i} \sim \mathcal{N}\left(0, \sigma^{2}\right)$ is independent and identically distributed (i.i.d.) over time, and $h_{i j}$ denotes the real channel from the $j$-th user to the $i$-th BS. In this paper, we assume that $X_{i}$ 's are real-valued Gaussian signals at fixed average powers $P_{i}$, for $i=1, \ldots, L$.
The notation used in this paper is as follows. Let $\mathbf{X}=$ $\left[X_{1}, X_{2}, \ldots, X_{L}\right]^{T}, \mathbf{Y}=\left[Y_{1}, Y_{2}, \ldots, Y_{L}\right]^{T}$. We use $\widehat{\mathbf{Y}}=$ $\left[\hat{Y}_{1}, \hat{Y}_{2}, \ldots, \hat{Y}_{L}\right]^{T}$ to denote the quantized signal vector. Let $\mathbf{H}$ be the $L \times L$ channel matrix with entries $h_{i j}$, and let $K_{X}=\mathbb{E}\left[\mathbf{X X}^{T}\right]$ denote the covariance matrix of the transmitted signal vector. Let $S \subseteq\{1,2, \ldots, L\}$. We use $\mathbf{X}(S)$ to denote the subset of $\mathbf{X}$ with elements whose indices are the elements of $S$. Let $q_{i}=\mathbb{E}\left(\hat{Y}_{i}-Y_{i}\right)^{2}$ be the average squarederror distortion between $Y_{i}$ and $\hat{Y}_{i}$. The distortion level $q_{i}$ is also referred to as the quantization noise level in this paper.

\section{V-MAC SCHEME WITH WYNER-ZIV COMPRESSION}

\section{A. Achievable Rate Region}

This paper uses the compress-and-forward scheme for the uplink MCP model under a sum backhaul constraint. As a BS cannot decode the transmitted messages by itself, it must quantize the received signal and transmit the quantization bits through its backhaul link to the CP. Because the received signals $\left\{Y_{i}\right\}_{i=1}^{L}$ at different BSs are statistically correlated, Wyner-Ziv compression can be employed to improve the efficiency of quantization. Further, this paper adopts a twostage strategy in which the CP first decodes the quantization codewords, and then decodes the user messages. More specifically, the $Y_{i}$ 's are first quantized at each BS using Wyner-Ziv compression. The compressed bits are sent to the $\mathrm{CP}$ through the noiseless links. The CP obtains the quantized signals $\widehat{\mathbf{Y}}$ using the quantized bits. The user messages are then decoded based on the quantized signals $\widehat{\mathbf{Y}}$. This scheme is referred to as the V-MAC-WZ scheme in this paper, because the users and the CP essentially form a virtual multiple-access channel. Note that this successive decoding scheme may in general achieve lower rates as compared to the approach of jointly decoding $\widehat{\mathbf{Y}}$ and $\mathbf{X}$ as in [4], [5] and [3, Corollary 1], but as shown later, successive decoding is already approximately optimal for sum capacity under the uplink MCP model considered in this paper.

Theorem 1: For the uplink MCP model with backhaul sum capacity constraint $C$ as shown in Fig. 1, the rate tuples $\left(R_{1}, R_{2}, \ldots, R_{L}\right)$ that satisfy the following set of constraints are achievable using the V-MAC-WZ scheme:

$$
\sum_{i \in S} R_{i} \leq \frac{1}{2} \log \frac{\left|\mathbf{H}_{S} K_{X(S)} \mathbf{H}_{S}^{T}+\Lambda_{q}+\sigma^{2} I\right|}{\left|\Lambda_{q}+\sigma^{2} I\right|}
$$

subject to

$$
\frac{1}{2} \log \frac{\left|\mathbf{H} K_{X} \mathbf{H}^{T}+\Lambda_{q}+\sigma^{2} I\right|}{\left|\Lambda_{q}\right|} \leq C
$$

for all $S \subseteq\{1,2, \ldots, L\}$, where $K_{X(S)}=E\left[\mathbf{X}(S) \mathbf{X}(S)^{T}\right]$ is the covariance matrix of $\mathbf{X}(S), \Lambda_{q}=\operatorname{diag}\left(q_{1}, q_{2}, \ldots, q_{L}\right)$, and $\mathbf{H}_{S}$ denotes the channel matrix from $\mathbf{X}(S)$ to $\mathbf{Y}$.

Proof: This theorem is a generalization of [3, Theorem 1], which treats the case of a single transmitter with multiple relays under individual backhaul capacity constraints. In [3, Theorem 1], it has been shown that $R<I(\mathbf{X} ; \widehat{\mathbf{Y}})$ is achievable subject to

$$
I\left(\mathbf{Y}(S) ; \widehat{\mathbf{Y}}(S) \mid \widehat{\mathbf{Y}}\left(S^{c}\right)\right) \leq \sum_{i \in S} C_{i}, \quad \forall S \subseteq\{1,2, \ldots, L\}
$$


under a product distribution $p(\hat{\mathbf{y}} \mid \mathbf{y})=\Pi_{i{ }_{1} 1}^{L} p\left(\hat{y}_{i} \mid y_{i}\right)$. Note that under the sum backhaul constraint $\sum_{i=1}^{\bar{L}} C_{i} \leq C$, the constraint (3) simply becomes $I(\mathbf{Y} ; \widehat{\mathbf{Y}}) \leq C$.

Now, with multiple users and considering the sum rate over any subset $S$, we likewise have

$$
\sum_{i \in S} R_{i} \leq I\left(\mathbf{X}(S) ; \hat{\mathbf{Y}} \mid \mathbf{X}\left(S^{c}\right)\right), \quad \forall S \subseteq\{1,2, \ldots, L\}
$$

subject to

$$
I(\mathbf{Y} ; \widehat{\mathbf{Y}}) \leq C
$$

Let $p\left(\hat{y}_{i} \mid y_{i}\right)$ be defined by the test channel $\hat{Y}_{i}=Y_{i}+Q_{i}$ where $Q_{i} \sim \mathcal{N}\left(0, q_{i}\right)$ and is independent of everything else. The achievable rate region (1) subject to (2) can now be derived by evaluating the mutual information expressions (4) and (5) using Gaussian distribution for the transmit signal $X_{i}$.

Note that the achievable rate in Theorem 1 depends on the transmit powers of the users. If users can dynamically change their transmit power levels, it is possible to further optimize the achievable rate over all possible transmit power levels. In this paper we assume that the transmit powers are fixed.

\section{B. Optimizing Quantization Noise Levels}

The achievable rate region (1)-(2) for the V-MAC-WZ scheme has an intuitive interpretation. The quantization process adds quantization noise to the overall multiple-access channel. Finer quantization results in higher overall rate, but also leads to higher backhaul capacity requirements. To characterize the tradeoff between the achievable rate and the backhaul constraint, it is important to optimize the achievable rate over the quantization noise levels $\left\{q_{1}, \ldots, q_{L}\right\}$.

In the following, we focus on the maximization of the overall sum rate under the sum backhaul capacity constraint. From Theorem 1, the optimization problem is

$$
\begin{array}{ll}
\text { maximize } & \frac{1}{2} \log \frac{\left|\mathbf{H} K_{X} \mathbf{H}^{T}+\sigma^{2} I+\Lambda_{q}\right|}{\left|\sigma^{2} I+\Lambda_{q}\right|} \\
\text { subject to } & \frac{1}{2} \log \frac{\left|\mathbf{H} K_{X} \mathbf{H}^{T}+\sigma^{2} I+\Lambda_{q}\right|}{\left|\Lambda_{q}\right|} \leq C \\
& \Lambda_{q}(i, j)=0, \text { for } i \neq j \\
& \Lambda_{q}(i, i)>0
\end{array}
$$

where $\Lambda_{q}(i, j)$ is the $(i, j)$-th entry of matrix $\Lambda_{q}$. Note that $\Lambda_{q}$ is diagonal because each BS quantizes its received signal independently.

The above optimization problem is nonconvex, thus finding its global optimum is challenging. However, the Karush-KuhnTucker (KKT) condition of the problem is still a necessary condition for optimality. To derive the KKT condition, we form the Lagrangian as follows:

$$
\begin{aligned}
L\left(\Lambda_{q}, \lambda, \Psi\right) & =(1-\lambda) \log \left|\mathbf{H} K_{X} \mathbf{H}^{T}+\sigma^{2} I+\Lambda_{q}\right| \\
& -\log \left|\sigma^{2} I+\Lambda_{q}\right|+\lambda \log \left|\Lambda_{q}\right|+\operatorname{tr}\left(\Psi \Lambda_{q}\right)
\end{aligned}
$$

where $\Psi$ is a matrix whose diagonal entries are zeros and the off-diagonal entries are the dual variables associated the constraint $\Lambda_{q}(i, j)=0$ for $i \neq j$, and $\lambda$ is the Lagrangian dual variable associated with the backhaul sum-capacity constraint. The coefficient $\frac{1}{2}$ is omitted for simplicity.

By setting $\partial L / \partial \Lambda_{q}$ to zero, we obtain the optimality condition

$$
\begin{aligned}
(1-\lambda)\left(\mathbf{H} K_{X} \mathbf{H}^{T}+\sigma^{2} I+\Lambda_{q}\right)^{-1} & -\left(\sigma^{2} I+\Lambda_{q}\right)^{-1} \\
& +\lambda \Lambda_{q}^{-1}+\Psi=0
\end{aligned}
$$

Recall that $\Psi$ has zeros on the diagonal, but can have arbitrary off-diagonal entries. Thus, the above optimality condition can be simplified as

$$
\begin{aligned}
(1-\lambda) \operatorname{diag}\left(\mathbf{H} K_{X} \mathbf{H}^{T}+\sigma^{2} I+\Lambda_{q}\right)^{-1} & -\left(\sigma^{2} I+\Lambda_{q}\right)^{-1} \\
& +\lambda \Lambda_{q}^{-1}=0
\end{aligned}
$$

We are now ready to derive the optimal quantization noise levels based on the above condition. Below, we first treat the high SNR case, then develop optimization algorithms for the general SNR case.

1) Optimal Quantization Noise Levels at High SNR: First, it is easy to verify that the optimality condition can only be satisfied if $0 \leq \lambda<1$. Second, since $\left(\Lambda_{q}+\sigma^{2} I\right)$ is the combined quantization and background noise, if the overall system is to operate at reasonably high spectral efficiency, we must have $\mathbf{H} K_{X} \mathbf{H}^{T} \gg \sigma^{2} I+\Lambda_{q}$. Under this high SNR condition, we have

$$
(1-\lambda) \operatorname{diag}\left(\mathbf{H} K_{X} \mathbf{H}^{T}+\sigma^{2} I+\Lambda_{q}\right)^{-1} \ll\left(\sigma^{2} I+\Lambda_{q}\right)^{-1}
$$

in which case the optimality condition becomes $\left(\sigma^{2} I+\right.$ $\left.\Lambda_{q}\right)^{-1}=\lambda \Lambda_{q}^{-1}$, or

$$
q_{i}^{*}=\frac{\lambda}{1-\lambda} \sigma^{2}
$$

where $\lambda \in[0,1)$ is chosen to satisfy the backhaul sum-capacity constraint $\frac{1}{2} \log \frac{\left|\mathbf{H} K_{X} \mathbf{H}^{T}+\sigma^{2} I+\Lambda_{q}\right|}{\left|\Lambda_{q}\right|}=C$. Note that $\lambda=0$ corresponds to the infinite backhaul case where $q_{i}^{*}=0$. As $\lambda$ increases, the sum backhaul capacity becomes increasingly constrained, and the optimal quantization noise level $q_{i}^{*}$ would also need to increase accordingly.

The main observation here is that under high SNR the quantization noise levels at different BSs should be chosen to be equal, regardless of the transmit power and the channel matrix. Uniform quantization noise levels are desirable in a practical implementation, because no adaptation to the channel condition is needed. This is especially advantageous in a fast fading environment.

2) Optimizing the Quantization Noise Levels under General SNR: In the general case where the SNR may not be high either because the background noise is comparable to the received signal, or because the sum backhaul capacity is severely constrained so that the entries of $\Lambda_{q}$ are large, we can still work with the optimality condition (9) to find the appropriate quantization noise levels.

Let $A_{i i}$ be the $i$-th diagonal element of the matrix $\left(\mathbf{H} K_{X} \mathbf{H}^{T}+\sigma^{2} I+\Lambda_{q}\right)^{-1}$. The optimality condition (9) states that

$$
(1-\lambda) A_{i i}+\frac{\lambda}{q_{i}}=\frac{1}{\sigma^{2}+q_{i}} .
$$


We can think of (11) as a fixed-point equation in $q_{i}$. In particular, we can ignore the dependence of $A_{i i}$ on $\left\{q_{1}, \ldots, q_{L}\right\}$, treat $A_{i i}$ as a constant, then solve the equation (11) for $q_{i}$. The fixed-point equation is quadratic in $q_{i}$, so it can be easily solved. In the event that multiple positive roots exist, we can select the $q_{i}$ that maximizes the Lagrangian (7).

The algorithm details are provided below, where $\Lambda_{q}^{(j)}$ and $A_{i i}^{(j)}$ denote the values of $\Lambda_{q}$ and $A_{i i}$ in the $j$-th iteration, respectively.

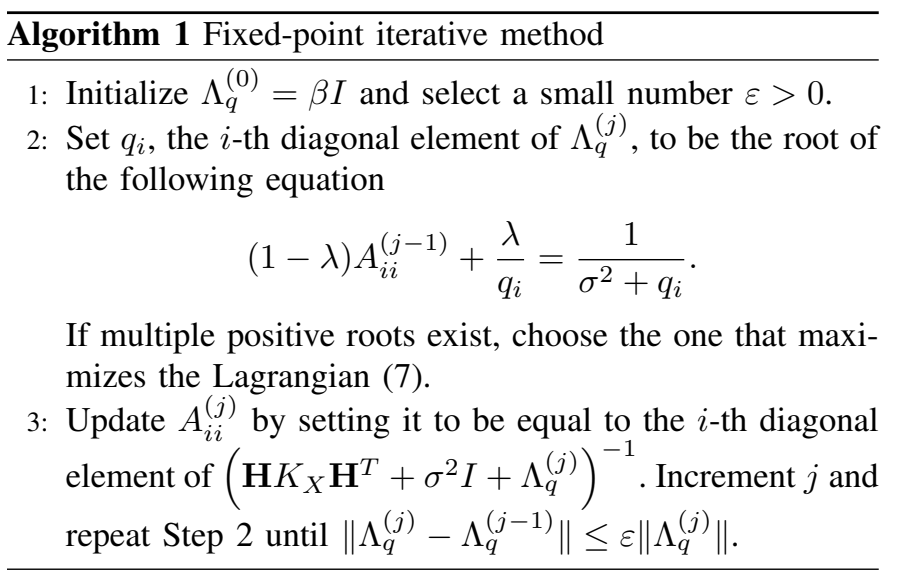

The above algorithm is found to converge faster than the algorithms in [8] for the single-antenna case considered in this papper. As the point of convergence satisfies the KKT condition, if the algorithm converges, it must reach a stationary point of problem (6). Although the stationary point is not guaranteed to be the globally optimum, simulation results suggest that the fixed-point algorithm works well in practice.

The dual variable $\lambda$ plays the role of constraining the backhaul sum capacity. In practice where a specific backhaul sumcapacity constraint must be met, it is possible to implement an efficient search over $\lambda$ in an outer loop.

The optimization of the quantization noise levels in this paper has the same flavor as the algorithms presented in [8], but with the key difference that this paper optimizes the quantization noise levels across all BSs jointly with a single objective of maximizing the sum rate, while the approaches in [8] iteratively optimize the quantization noise level of one BS at a time in a coordinated fashion, where the objective function in each step is the maximization of the achievable rate for one BS only. For this reason, the joint optimization algorithm in this paper is better suited for the sum-rate maximization.

\section{Sum Capacity to Within a Constant Gap}

One of the key insights from the previous section is that the quantization noise levels across the BSs should be set to be approximately uniform. In this section, we further justify this choice of uniform quantization noise levels by showing that doing so in fact achieves the sum capacity of the uplink MCP model with sum backhaul capacity constraint to within a constant gap. The gap depends on the number of BSs in the network but is independent of the channel matrix and the SNRs. The main result of this section is the following:
Theorem 2: For the uplink MCP model with $L$ base-stations and sum backhaul capacity $C$ shown in Fig. 1, the V-MACWZ scheme with uniform quantization noise levels across the BSs (i.e., $q_{i}=q$ ) achieves the sum capacity to within $\frac{L}{2}$ bits.

Proof: The idea is to choose $q_{i}=\alpha \sigma^{2}, i=1,2, \ldots, L$ where $\alpha>0$ is an appropriately chosen constant, then compare the achievable rate of V-MAC-WZ with the following cut-set like sum-capacity upper bound [4]

$$
\bar{C}=\min \left\{\frac{1}{2} \log \frac{\left|\mathbf{H} K_{X} \mathbf{H}^{T}+\sigma^{2} I\right|}{\left|\sigma^{2} I\right|}, C\right\}
$$

where the first term is the cut from the users to the basestations, and the second term is the cut across the backhaul.

We choose the quantization level $\alpha$ depending on $C$ as follows: When $C \geq \frac{1}{2} \log \frac{\left|\mathbf{H} K_{X} \mathbf{H}^{T}+2 \sigma^{2} I\right|}{\left|\sigma^{2} I\right|}$, we choose $\alpha=1$, i.e., the quantization levels are set to be at the background noise level. Since $\alpha=1$, it can be verified that

$$
I(\mathbf{Y} ; \widehat{\mathbf{Y}})=\frac{1}{2} \log \frac{\left|\mathbf{H} K_{X} \mathbf{H}^{T}+2 \sigma^{2} I\right|}{\left|\sigma^{2} I\right|} .
$$

Thus, we have $C \geq I(\mathbf{Y} ; \widehat{\mathbf{Y}})$. This implies that the sum backhaul constraint (2) is satisfied. Therefore, the sum rate

$$
R_{\text {sum }}=I(\mathbf{X} ; \widehat{\mathbf{Y}})=\frac{1}{2} \log \frac{\left|\mathbf{H} K_{X} \mathbf{H}^{T}+2 \sigma^{2} I\right|}{\left|2 \sigma^{2} I\right|}
$$

is achievable. In this case, the gap between $\bar{C}$ and $R_{\text {sum }}$ can be bounded by

$$
\begin{aligned}
\bar{C}-R_{\text {sum }} \leq & \frac{1}{2} \log \frac{\left|\mathbf{H} K_{X} \mathbf{H}^{T}+\sigma^{2} I\right|}{\left|\sigma^{2} I\right|} \\
& -\frac{1}{2} \log \frac{\left|\mathbf{H} K_{X} \mathbf{H}^{T}+2 \sigma^{2} I\right|}{\left|2 \sigma^{2} I\right|}<\frac{L}{2}
\end{aligned}
$$

When $C<\frac{1}{2} \log \frac{\left|\mathbf{H} K_{X} \mathbf{H}^{T}+2 \sigma^{2} I\right|}{\left|\sigma^{2} I\right|}$, we choose $\alpha$ so that $I(\mathbf{Y} ; \widehat{\mathbf{Y}})=C$. First, note that for such a choice of $\alpha$ the sum rate $R_{\text {sum }}=I(\mathbf{X} ; \widehat{\mathbf{Y}})$ is achievable. Next, observe that

$$
I(\mathbf{Y} ; \widehat{\mathbf{Y}})=\frac{1}{2} \log \frac{\left|\mathbf{H} K_{X} \mathbf{H}^{T}+\sigma^{2} I+\alpha \sigma^{2} I\right|}{\left|\alpha \sigma^{2} I\right|}
$$

is a monotonically decreasing function of $\alpha$. Since $C=$ $I(\mathbf{Y} ; \widehat{\mathbf{Y}})<\frac{1}{2} \log \frac{\left|\mathbf{H} K_{X} \mathbf{H}^{T}+2 \sigma^{2} I\right|}{\left|\sigma^{2} I\right|}$, we have $\alpha>1$. Now, we use $C=I(\mathbf{Y} ; \widehat{\mathbf{Y}})$ as an upper bound. The gap between $\bar{C}$ and $R_{\text {sum }}$ can now be bounded by

$$
\begin{aligned}
\bar{C}-R_{\text {sum }} \leq & I(\mathbf{Y} ; \widehat{\mathbf{Y}})-I(\mathbf{X} ; \widehat{\mathbf{Y}}) \\
= & \frac{1}{2} \log \frac{\left|\mathbf{H} K_{X} \mathbf{H}^{T}+(1+\alpha) \sigma^{2} I\right|}{\left|\alpha \sigma^{2} I\right|} \\
& -\frac{1}{2} \log \frac{\left|\mathbf{H} K_{X} \mathbf{H}^{T}+(1+\alpha) \sigma^{2} I\right|}{\left|(1+\alpha) \sigma^{2} I\right|} \\
= & \frac{L}{2} \log \left(1+\frac{1}{\alpha}\right)<\frac{L}{2}
\end{aligned}
$$

where the last inequality follows from the fact that $\alpha>1$.

Combining the two cases, we see that the gap to the sum capacity for the V-MAC-WZ scheme with appropriately 
chosen quantization noise levels (which are uniform across the BSs) is always less than $\frac{L}{2}$.

Theorem 2 demonstrates that when $C$ is sufficiently large, the quantization noise levels can essentially be set to be at the background noise level, resulting in a gap to the cut-set bound of at most $1 / 2$ bits per dimension per user. When $C$ is small, the quantization noise levels across all the BSs can be scaled uniformly, while maintaining the same gap. The constant gapto-optimality result depends on the particular feature of the sum-capacity constrained backhaul, and it does not hold in the case of individual backhaul constraints [3], [4].

\section{Simulation Results}

To demonstrate the effectiveness of the proposed quantization noise level optimization algorithm, we evaluate the achievable sum rates of the uplink MCP model using different choices of $q_{i}$ in a realistic 7-cell wireless network with one user per cell. Standard cellular network parameters are used in the numerical evaluation: the noise power spectral density (PSD) is set to be $-169 \mathrm{dBm} / \mathrm{Hz}$; the users' transmit power is set to be $23 \mathrm{dBm}$; frequency selective channels are used according to a pedestrian multi-path time delay profile, a distancedependent path-loss model $L=128.1+37.6 \cdot \log _{10}(d)$, (where $d$ is the distance in $\mathrm{km}$ ), and with $8 \mathrm{~dB} \log$ normal shadowing and a Rayleigh component. The distance between neighboring base-stations is set to be $0.5 \mathrm{~km}$.

The numerical results are shown in Fig. 2, where

- the uniform quantization noise levels refer to $q_{i}=\lambda /(1-$ त) $\sigma^{2}$ as given by (10) assuming high SNR;

- the approximately optimal quantization noise levels are those used in the proof of Theorem 2;

- the optimized quantization noise levels are the $q_{i}$ 's produced by the fixed-point iteration algorithm.

It is verified that the achievable sum rate per cell when using the approximately optimal quantization noise levels is indeed less than $\frac{L}{2}=\frac{7}{2}$ bits away from the sum-capacity upper bound. However, it is a poor choice of quantization noise levels when $C$ is large, because the quantization noise levels are set to be at the background noise level, and are not reduced further as the available sum backhaul capacity $C$ grows.

Moreover, for all values of $C$, using uniform quantization noise levels is almost as good as using the optimized quantization noise levels produced by the fixed-point iteration algorithm. Both the uniform and the optimized quantization noise levels produce a graceful tradeoff between the sum backhaul capacity and the achievable sum rate. Overall, the numerical simulation demonstrates that choosing uniform quantization noise levels is near optimal.

\section{CONCLUSION}

This paper studies an uplink MCP model where the BSs are connected to a $\mathrm{CP}$ through noiseless backhaul links of limited sum capacity. We utilize a so-called V-MAC-WZ scheme, where the quantization codewords are first decoded at the $\mathrm{CP}$; subsequently the users are treated as if they form a virtual multiple-access channel. This paper shows that under the

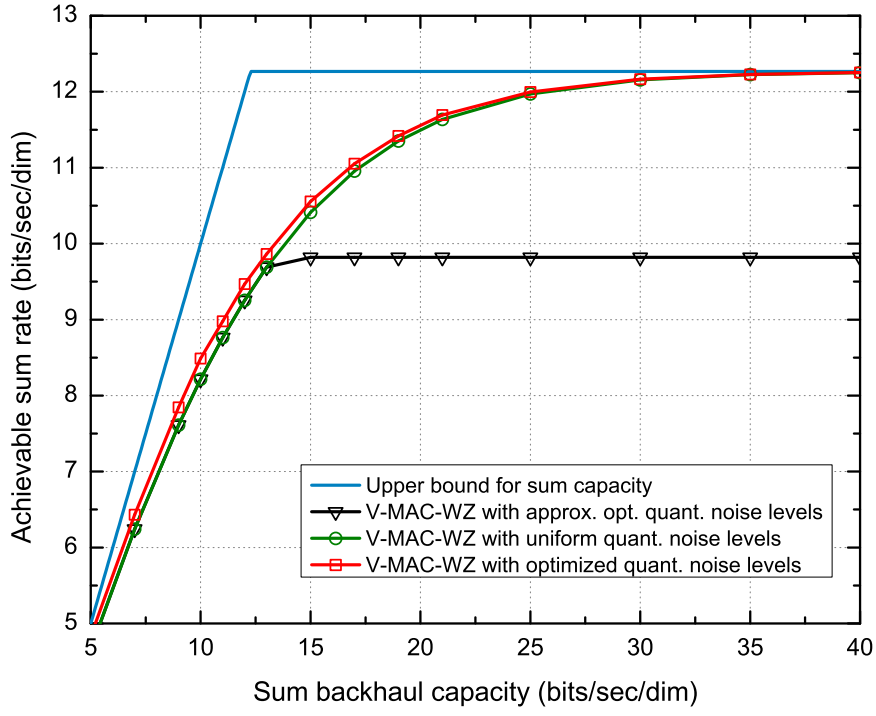

Fig. 2. Achievable sum rates of the V-MAC-WZ scheme with different choices of quantization noise levels for a 7 -cell wireless network.

proposed model, choosing uniform quantization levels across all the BSs is near optimal at high SNR. We further develop a numerical algorithm for optimizing the quantization noise levels. Finally, we show that the V-MAC-WZ scheme achieves the sum capacity of the uplink MCP model to within a constant gap that only depends on the number of BSs.

The results of this paper demonstrate several key advantages of the V-MAC-WZ scheme: the quantization noise levels can be readily optimized, and it avoids the complexity of joint decoding by performing successive decoding, while providing near optimal performance over a range of channel conditions and backhaul constraints.

\section{REFERENCES}

[1] D. Gesbert, S. Hanly, H. Huang, S. Shamai, O. Simeone, and W. Yu, "Multi-cell MIMO cooperative networks: A new look at interference," IEEE J. Sel. Areas Commun., vol. 28, no. 9, pp. 1380-1408, Dec. 2010.

[2] P. Marsch and G. Fettweis, "Uplink CoMP under a constrained backhaul and imperfect channel knowledge," IEEE Trans. Wireless Commun., vol. 10, no. 6, pp. 1730-1742, June 2011.

[3] A. Sanderovich, S. Shamai, Y. Steinberg, and G. Kramer, "Communication via decentralized processing," IEEE Trans. Inf. Theory, vol. 54, no. 7, pp. 3008-3023, July 2008.

[4] A. Sanderovich, O. Somekh, H. V. Poor, and S. Shamai, "Uplink macro diversity of limited backhaul cellular network," IEEE Trans. Inf. Theory, vol. 55, no. 8, pp. 3457-3478, Aug. 2009.

[5] A. Sanderovich, S. Shamai, and Y. Steinberg, "Distributed MIMO receiver - Achievable rates and upper bounds," IEEE Trans. Inf. Theory, vol. 55, no. 10, pp. 4419-4438, Oct. 2009.

[6] A. Avestimehr, S. Diggavi, and D. Tse, "Wireless network information flow: A deterministic approach," IEEE Trans. Inf. Theory, vol. 57, no. 4, pp. 1872-1905, Apr. 2011.

[7] S. H. Lim, Y.-H. Kim, A. El Gamal, and S.-Y. Chung, "Noisy network coding," IEEE Trans. Inf. Theory, vol. 57, no. 5, pp. 3132-3152, May 2011.

[8] A. del Coso and S. Simoens, "Distributed compression for MIMO coordinated networks with a backhaul constraint," IEEE Trans. Wireless Commun., vol. 8, no. 9, pp. 4698-4709, Sept. 2009.

[9] S.-H. Park, O. Simeone, O. Sahin, and S. Shamai, "Robust and efficient distributed compression for cloud radio access networks," IEEE Trans. Veh. Technol., vol. 62, no. 2, pp. 692-703, Feb. 2013. 